\title{
Efficacy and Safety of Ixekizumab with or Without Methotrexate in Biologic-Naïve Patients with Psoriatic Arthritis: 52-Week Results from SPIRIT-H2H Study
}

\author{
Josef S. Smolen · Anthony Sebba · Eric M. Ruderman • \\ Hendrik Schulze-Koops - Christophe Sapin · Amanda M. Gellett • \\ Aubrey Trevelin Sprabery · Lingnan Li · Inmaculada de la Torre • \\ Gaia Gallo · Soyi Liu-Leage · Sreekumar Pillai · Paulo Reis • \\ Peter Nash
}

Received: September 11, 2020 / Accepted: October 26, 2020 / Published online: November 16, 2020

(C) The Author(s) 2020

\section{ABSTRACT}

Introduction: In the SPIRIT-H2H (ClinicalTrials.gov: NCT03151551) trial in biologic-naïve patients with active psoriatic arthritis (PsA), ixekizumab (IXE) was superior to adalimumab

Electronic supplementary material The online version of this article (https://doi.org/10.1007/s40744020-00250-3) contains supplementary material, which is available to authorized users.

J. S. Smolen

Division of Rheumatology, Department of Medicine III, Medical University of Vienna, Vienna, Austria

A. Sebba

Arthritis Associates, Palm Harbor, FL, USA

E. M. Ruderman

Northwestern University Feinberg School of

Medicine, Chicago, IL, USA

H. Schulze-Koops

Division of Rheumatology and Clinical

Immunology, Department of Internal Medicine IV, Ludwig-Maximilians University of Munich, Munich, Germany

C. Sapin · A. M. Gellett - A. T. Sprabery · L. Li ·

I. de la Torre · G. Gallo - S. Liu-Leage - S. Pillai ·

P. Reis

Eli Lilly and Company, Indianapolis, IN, USA

P. Nash $(\square)$

School of Medicine, Griffith University, Brisbane, QLD, Australia

e-mail: drpnash@tpg.com.au
(ADA) at week 24 in terms of achieving a combined endpoint of $\geq 50 \%$ improved response in the American College of Rheumatology scale score (ACR50) and 100\% improvement in the Psoriasis Areas and Severity Index (PASI100), and was non-inferior in terms of achieving ACR50. IXE resulted in similar improvements of PsA manifestations irrespective of the use of concomitant conventional synthetic diseasemodifying anti-rheumatic drugs (csDMARDs), while ADA response was higher with concomitant csDMARD use. The aim of this study was to determine the efficacy and safety of treatment with IXE and ADA with or without methotrexate (MTX), the most commonly use csDMARD, through week 52 in patients with PsA.

Methods: In the open-label, rater-blinded, head-to-head SPIRIT-H2H trial, randomization of patients was stratified by concomitant use of csDMARD and moderate-to-severe plaque psoriasis involvement. In the post-hoc subgroup analysis presented here, subgroups were defined as with/without concomitant MTX use at baseline. Treatment group effects within subgroups were tested using Fisher's exact test. Missing data were imputed using non-responder imputation.

Results: By week 52, IXE provided similar improvements in the combined ACR50 and PASI100 endpoint, ACR50, and other PsA-related domains regardless of whether IXE was used with or without MTX, while ADA efficacy 
appeared to be improved with concomitant MTX use. When used without concomitant MTX, IXE resulted in significantly higher response versus ADA in terms of the combined ACR50 and PASI100 $(p=0.002)$ endpoint, minimal disease activity $(p=0.016)$, and very low disease activity $(p=0.037)$. The safety of both agents was consistent with their known safety profiles regardless of concomitant MTX use.

Conclusion: In PsA patients with inadequate control of the disease, IXE delivers consistent efficacy in several clinical domains of the disease regardless of concomitant MTX use. The efficacy of ADA is increased by the concomitant use of MTX. These findings can inform treatment decisions when considering the need for concomitant MTX use with IXE or ADA at initiation or for long-term maintenance.

Keywords: Adalimumab; CsDMARD; Ixekizumab; Methotrexate; Psoriatic arthritis

\section{Key Summary Points}

\section{Why carry out this study?}

Limited evidence is currently available on the impact of concomitant use of methotrexate (MTX) on the efficacy and safety of biologic disease-modifying antirheumatic drugs (bDMARDs) approved for the treatment of patients with psoriatic arthritis (PsA).

The aim of this study was to investigate the efficacy and safety of two bDMARDs, ixekizumab and adalimumab, with or without MTX through week 52 in patients with PsA.

\section{What was learned from the study?}

The results of this study suggest that ixekizumab demonstrated identical efficacy in joint endpoints with or without concomitant MTX, while adalimumab efficacy appears to increase with the concomitant use of MTX.
The safety of both agents was consistent with their known safety profiles regardless of concomitant MTX use.

The results of this study inform evidencebased treatment decisions when considering concomitant MTX use when prescribing ixekizumab or adalimumab for patients with PsA.

\section{DIGITAL FEATURES}

This article is published with digital features, including a summary slide, to facilitate understanding of the article. To view digital features for this article go to https://doi.org/10.6084/ m9.figshare.13135763.

\section{INTRODUCTION}

Psoriatic arthritis (PsA) is a heterogeneous inflammatory disease [1-3] that is characterized by the presence of arthritis and often also by other musculoskeletal manifestations, including enthesitis, dactylitis, and/or axial involvement, potentially resulting in joint damage, impaired physical function, and reduced quality of life (QoL). In addition to joint symptoms, PsA is often associated with extra-articular manifestations, including skin and nail psoriasis that contribute to the increased burden of illness and the reduced QoL $[1,4]$.

Treatment for PsA remains challenging due to the heterogeneity of the disease. The European League Against Rheumatism (EULAR) 2019 recommendations include the initial use of non-steroidal anti-inflammatory drugs for musculoskeletal manifestations followed by single conventional synthetic disease-modifying anti-rheumatic drugs (csDMARDs), such as methotrexate (MTX), which is the most frequently used csDMARD in PsA [5, 6]. While data on the efficacy of MTX in monotherapy are controversial [7], recent studies, such as the 
TICOPA and PsA-SEAM trials, reveal a high level of efficacy $[8,9]$, and some even interpret the efficacy as being similar to that of a tumor necrosis factor inhibitor (TNFi) [10].

The beneficial effect of the concomitant use of MTX with a biologic DMARD (bDMARD) in patients with PsA is still being debated [11]. The EULAR recommends using bDMARDs, such as TNFi and anti-interleukin (IL)-12/23 or IL-17A antagonists, as add-on to csDMARD or as a monotherapy for PsA patients with inadequate response to csDMARDs. There is limited evidence to support the clinical decision of whether to use concomitant MTX with bDMARDs from the beginning of a patient's care or whether to maintain or stop concomitant MTX in patients with PsA with a good clinical response [12]. Although the TEMPO, PREMIER, GOAFTER, SURPRISE and ACT-RAY studies conducted in patients with rheumatoid arthritis have shown better efficacy with bDMARDs when combined with MTX compared with bDMARD monotherapy [13-17], data comparing combination therapy with bDMARD monotherapy in patients with PsA are limited and inconclusive [9].

Ixekizumab (IXE) is a high-affinity monoclonal antibody that selectively targets IL-17A and is approved for the treatment of moderateto-severe plaque psoriasis (PsO), PsA, ankylosing spondylitis, non-radiographic axial spondylarthritis, and pediatric PsO [18-21]. SPIRIT$\mathrm{H} 2 \mathrm{H}$ (ClinicalTrials.gov: NCT03151551) is a 52-week trial evaluating the efficacy and safety of IXE versus adalimumab (ADA), a TNFi, in bDMARD-naïve patients with active PsA and inadequate response to csDMARDs. IXE was found to be superior to ADA at week 24 for the combined improvement in joint and skin disease (as measured by the percentage of patients achieving $\geq 50 \%$ improvement in the American College of Rheumatology scale score [ACR50] and 100\% improvement from baseline in the Psoriasis Areas and Severity Index rating score [PASI100]) [22]. Furthermore, IXE achieved the major secondary endpoints at week 24 of non-inferiority of IXE versus ADA for ACR50 responses and superiority of IXE versus ADA for PASI100 response. Also, a greater proportion of patients receiving IXE compared with ADA achieved the combined endpoints of ACR50 and PASI100 response at week 52. For other musculoskeletal-related domains, the responses observed at week 24 were maintained until week 52. IXE efficacy on the combined ACR50 and PASI100 response, as well as on separately assessed ACR50 and PASI100 responses, were similar with or without csDMARDs, while response to ADA seemed better with concomitant csDMARD use [23].

The objective of the current analysis is to evaluate the efficacy of IXE and ADA through 52 weeks of treatment when used with and without concomitant MTX.

\section{METHODS}

\section{Participants}

Inclusion criteria have been detailed in a previous publication [22]. Briefly, eligible patients were aged $\geq 18$ years, had an established diagnosis of PsA for at least 6 months, fulfilled the ClASsification for Psoriatic ARthritis (CASPAR) criteria, and had active PsA defined as at least $3 / 66$ swollen and at least 3/68 tender joints. Patients were also required to have active $\mathrm{PsO}$ affecting $\geq 3 \%$ of body surface area (BSA), had previous inadequate response to $\geq 1 \mathrm{csDMARD}$, and had no prior treatment with any bDMARD or targeted synthetic DMARDS.

\section{Study Design and Treatment}

The SPIRIT-H2H study design has been described in a previous publication [22]. Briefly, it is a phase $3 \mathrm{~b} / 4$, multicenter, randomized, open-label, parallel-group (head-to-head) trial with a 52-week duration with rater-blinded outcome assessments evaluating the efficacy and safety of IXE versus ADA in bDMARD-naïve patients with PsA and active PsO who had previous history of inadequate response to csDMARDs. During a 52-week open-label treatment period (weeks $0-52$ ), patients were randomly assigned in a 1:1 ratio to receive subcutaneous injections of either IXE $80 \mathrm{mg}$ every 4 weeks starting at week 4 or a 40-mg starting dose of $\mathrm{ADA}$ at 
randomization (week 0) followed by $40 \mathrm{mg}$ ADA every 2 weeks starting at week 2 (unless they met the criteria for moderate-to-severe PsO). Patients with moderate-to-severe $\mathrm{PsO}$ received either $80 \mathrm{mg}$ IXE every 2 weeks from week 2 to week 12 , followed by $80 \mathrm{mg}$ IXE every 4 weeks or an $80 \mathrm{mg}$ starting dose of ADA (two injections) at week 0 , followed by $40 \mathrm{mg}$ ADA every 2 weeks starting at week 1 . For all patients assigned to the IXE treatment arm, the starting dose was $160 \mathrm{mg}$ (two $80 \mathrm{mg}$ subcutaneous injections) administrated at randomization. Randomization was stratified by concomitant csDMARD use at baseline and moderate-to-severe $\mathrm{PsO}$ involvement (PASI $\geq 12, \mathrm{BSA} \geq 10 \%$ and Static Physician's Global Assessment [sPGA] $\geq$ 3). MTX, when included, must have been used for at least 12 weeks and as a stable dose for at least 8 weeks prior to randomization. The permitted MTX dose was $10-25 \mathrm{mg} /$ week oral or parenteral. Changes in MTX background use were not permitted during the first 24 weeks of the study period except for safety reasons. Adjustment in MTX background use was allowed after week 24 .

All procedures performed in studies involving human participants were in accordance with the ethical standards of the institutional and/or national research committees at all sites where these studies were conducted and with the 1964 Helsinki declaration and its later amendments or comparable ethical standards. Informed consent was obtained from all individual participants included in the studies. The study protocol was approved by the ethical review board of all participating centers prior to the start of study-related procedures (see Electronic Supplementary Material [ESM] for the full list of ethics committees). The main ethics committee was Whipps Cross University Hospital, London, UK (registration no. 17/LO/ 0794).

\section{Efficacy and Safety Endpoints}

Endpoints were assessed in subgroups defined by the presence or absence of concomitant MTX use at baseline (post-hoc subgroups). The primary endpoint was the proportion of patients who achieved combined ACR50 and PASI100 responses at week 24. After the week-24 database lock and initial analysis run, a medical inconsistency in baseline PASI data was identified. Although nine patients were assessed as PASI $=0$ at baseline (including 5 patients with MTX at baseline and 4 patients without MTX at baseline), those patients fulfilled the criteria for having psoriasis as assessed by a BSA $\geq 3 \%$. Therefore, those patients were judged as being PASI100 responders if they achieved an absolute PASI $=0$ and $\mathrm{BSA}=0$ at post-baseline visits. Additional prespecified outcomes assessed the proportion of patients achieving 20, 50 or $70 \%$ improvement from baseline in the ACR scale score (ACR20/50/70, respectively); resolution of enthesitis as measured by the Spondyloarthritis Research Consortium of Canada Enthesitis Index (SPARCC Enthesitis Index $=0$ ) or Leeds Enthesitis Index $(\mathrm{LEI}=0)$ among patients with enthesitis at baseline (SPARCC Enthesitis Index $>0$ or LEI $>0$, respectively); resolution of dactylitis as measured by the Leeds Dactylitis Index-Basic (LDI-B =0) among patients with dactylitis at baseline (LDI-B $>0$ ); a modified Composite Psoriatic Disease Activity Index (mCPDAI) $\leq 5$ (low disease activity or remission); $\geq 75 \%, \geq 90 \%$, or $\geq 100 \%$ improvement from baseline in PASI (PASI75/90/100, respectively); resolution of fingernail psoriasis (Nail Psoriasis Area and Severity Index [NAPSI] fingernails $=0)$; PsA Dermatology Life Quality Index score of 0 or 1 (DLQI $[0,1]$ ); and minimal disease activity (MDA) assessed by 18 entheseal points (MDA was achieved if patients fulfilled 5 of 7 outcome measures: tender joint count $\leq 1$; swollen joint count $\leq 1$; PASI total score $\leq 1$ or $\mathrm{BSA} \leq 3$; patient pain visual analog scale (VAS) score of $\leq 15$; patient global assessment VAS score of $\leq 20$; Health Assessment Questionnaire-Disability Index (HAQ-DI) score of $\leq 0.5$; and tender entheseal points $\leq 1)$. Other outcomes included the proportion of patients achieving Disease Activity in Psoriatic Arthritis (DAPSA) score $\leq 4$ (remission) and DAPSA $\leq 14$ (low disease activity or remission) and very low disease activity (VLDA).

Treatment-emergent adverse events (TEAEs) were defined as an event that first occurred or worsened in severity after baseline (first dose), 
on or before the last day of the treatment period. General safety included TEAEs, serious adverse events (SAEs), deaths, and discontinuations due to adverse events (AEs). AEs of special interest included infections, serious infections, malignancies, major adverse cerebrocardiovascular events (MACE), inflammatory bowel disease, injection-site reactions, depression, hepatic laboratory changes, cytopenia, and neutropenia.

\section{Efficacy and Safety Analyses}

Post-hoc analyses of efficacy and QoL outcomes were performed through week 52 in the intentto-treat population, consisting of all randomized patients according to treatment assigned at week 0 . For the efficacy analysis, treatment group differences were evaluated within each MTX subgroup using Fisher's exact test. Missing data were imputed using non-responder imputation. Any $p$ value below 0.05 was considered to be statistically significant.

The study was not powered to perform statistical comparison of safety data between two treatment arms. Descriptive statistics were performed on the safety population, defined as all randomized patients who received $\geq 1$ dose of the study treatment.

\section{RESULTS}

\section{Participants}

Patient demographic and baseline characteristics are provided in Table 1 . Of the 566 patients (intent-to-treat population) with PsA selected for this post-hoc analysis, 283 patients received IXE (116 without MTX and 167 with MTX) and 283 patients received ADA (114 without MTX and 169 with MTX). Among patients taking MTX, 9.0\% (15/167) and 7.1\% (12/169) treated with IXE and ADA, respectively, were taking an additional csDMARD (leflunomide, sulfasalazine, or cyclosporine). Baseline demographics and disease characteristics were well balanced between treatment groups.

\section{Efficacy}

\section{Major Outcomes}

Efficacy outcomes at week 52 are summarized in Table 2. At week 52, combined ACR50 and PASI100 response (primary endpoint) with IXE was similar regardless of whether it was used with $(38.9 \%)$ or without $(39.7 \%)$ MTX. ADA response was numerically higher when used with MTX (30.2\%) than without MTX (20.2\%). For patients not taking MTX, significantly $(p=0.002)$ more patients achieved the primary endpoint with IXE (39.7\%) than with ADA (20.2\%); significant differences were observed as early as week 8 (Fig. 1a). For patients taking concomitant MTX, a numerically higher proportion of patients treated with IXE achieved the primary endpoint compared with those taking ADA (IXE 38.9\%, ADA 30.2\%, $p=0.108$; Fig. 1b). ACR50 response was similar when IXE was used with (47.9\%) or without (52.6\%) MTX, while ADA response was numerically higher when the treatment was used with (56.2\%) than without (40.4\%) MTX (Table 2). There were no statistically significant differences in ACR50 response between treatment arms when used with or without MTX throughout the 52 weeks observed (Fig. 1c, d).

\section{PsA Treatment Targets According to Treat-to- Target}

At week 52, MDA response in patients treated with IXE was similar regardless of whether it was used with (46.7\%) or without (48.3\%) MTX, while ADA response was numerically higher when used with MTX (46.7\%) than without MTX (32.5\%). When used without MTX, significantly more patients achieved MDA at week 52 in the IXE (48.3\%) versus ADA (32.5\%) group $(p=0.016)$ (Table 2; Fig. 2a). Among patients using MTX, similar responses in terms of MDA were observed between treatment groups at week 52 (Fig. 2b). VLDA response with IXE was numerically lower when used with MTX $(20.4 \%)$ than without MTX (27.6\%), whereas ADA efficacy of VLDA was numerically higher with MTX (21.3\%) than without MTX (15.8\%). For patients not taking MTX, significantly $(p=0.037)$ more patients achieved VLDA with 
Table 1 Baseline demographics and disease characteristics

\begin{tabular}{|c|c|c|c|c|}
\hline \multirow{2}{*}{$\begin{array}{l}\text { Baseline demographics and disease } \\
\text { characteristics }\end{array}$} & \multicolumn{2}{|c|}{ IXE treatment arm } & \multicolumn{2}{|c|}{ ADA treatment arm } \\
\hline & $\begin{array}{l}\text { No MTX } \\
(N=116)\end{array}$ & $\begin{array}{l}\text { MTX } \\
(N=167)\end{array}$ & $\begin{array}{l}\text { No MTX } \\
(N=114)\end{array}$ & $\begin{array}{l}\text { MTX } \\
(N=169)\end{array}$ \\
\hline \multicolumn{5}{|l|}{ Baseline demographics } \\
\hline Age (years) & $47.3(12.2)$ & $47.7(11.9)$ & $49.9(13.2)$ & $47.1(11.6)$ \\
\hline \multicolumn{5}{|l|}{ Sex, $n(\%)$} \\
\hline Male & $65 / 116(56.0)$ & $97 / 167(58.1)$ & $60 / 114(52.6)$ & $90 / 169(53.3)$ \\
\hline \multicolumn{5}{|l|}{ Race, $n(\%)$} \\
\hline White & $96 / 116(82.8)$ & $\begin{array}{r}126 / 167 \\
(75.4)\end{array}$ & $102 / 114(89.5)$ & $\begin{array}{r}109 / 169 \\
(64.5)\end{array}$ \\
\hline Asian & $5 / 116(4.3)$ & 24/167 (14.4) & $2 / 114(1.8)$ & $31 / 169(18.3)$ \\
\hline Body mass index $\left(\mathrm{kg} / \mathrm{m}^{2}\right)$ & $29.8(6.2)$ & $30.2(7.3)$ & $29.7(6.0)$ & $29.7(9.5)$ \\
\hline $\begin{array}{l}\text { Duration of symptoms since PsA diagnosis } \\
\text { (years) }\end{array}$ & $7.1(7.5)$ & $6.2(7.3)$ & $6.1(6.2)$ & $5.8(6.4)$ \\
\hline $\begin{array}{l}\text { Duration of symptoms since psoriasis diagnosis } \\
\text { (years) }\end{array}$ & $16.9(13.2)$ & $15.5(13.1)$ & $17.3(13.0)$ & $13.0(12.0)$ \\
\hline Mean concomitant MTX dosage (mg/week) & 0 & $16.6(4.1)$ & 0 & $16.5(4.6)$ \\
\hline \multicolumn{5}{|l|}{ Disease characteristics } \\
\hline Tender joint count & $19.2(13.5)$ & $19.0(12.1)$ & $24.0(17.8)$ & $19.5(13.2)$ \\
\hline Swollen joint count & $10.1(7.4)$ & $10.1(7.6)$ & $12.5(10.3)$ & $9.4(5.9)$ \\
\hline Patient's assessment of joint pain VAS (mm) & $58.4(21.6)$ & $60.6(22.2)$ & $63.0(22.1)$ & $61.9(20.4)$ \\
\hline $\begin{array}{l}\text { Patient's global assessment of disease activity } \\
\text { VAS }(\mathrm{mm})\end{array}$ & $62.7(21.9)$ & $62.2(19.2)$ & $66.4(21.6)$ & $64.3(20.2)$ \\
\hline $\begin{array}{l}\text { Physician's global assessment of disease activity } \\
\text { VAS (mm) }\end{array}$ & $59.4(18.2)$ & $58.6(17.1)$ & $60.6(20.0)$ & $58.6(16.9)$ \\
\hline HAQ-DI total score & $1.1(0.6)$ & $1.3(0.6)$ & $1.2(0.7)$ & $1.3(0.7)$ \\
\hline C-reactive protein $(\mathrm{mg} / \mathrm{L})$ & $8.5(13.2)$ & $10.8(13.9)$ & $10.6(22.2)$ & $10.5(17.1)$ \\
\hline SPARCC Enthesitis Index $>0, n(\%)$ & $78 / 115(67.8)$ & $\begin{array}{r}111 / 167 \\
(66.5)\end{array}$ & $76 / 114(66.7)$ & $95 / 169(56.2)$ \\
\hline SPARCC Enthesitis Index ${ }^{a}$ & $5.5(3.9)$ & $4.5(3.1)$ & $6.1(3.8)$ & $5.4(3.7)$ \\
\hline LEI $>0, n(\%)$ & $67 / 115(58.3)$ & $92 / 167(55.1)$ & $67 / 114(58.8)$ & $80 / 169(47.3)$ \\
\hline $\mathrm{LEI}^{\mathrm{b}}$ & $2.9(1.5)$ & $2.2(1.2)$ & $2.9(1.5)$ & $2.6(1.5)$ \\
\hline LDI-B $>0, n(\%)$ & $18 / 115(15.7)$ & $24 / 167(14.4)$ & $26 / 114(22.8)$ & $32 / 169(18.9)$ \\
\hline LDI-B $^{c}$ & $48.4(40.4)$ & $33.8(43.5)$ & $80.9(188.1)$ & $35.4(28.6)$ \\
\hline Moderate-to-severe psoriasis, $n$ (\%) & $24 / 116(20.7)$ & $25 / 167(15.0)$ & $23 / 114(20.2)$ & $28 / 168(16.7)$ \\
\hline
\end{tabular}


Table 1 continued

\begin{tabular}{llllll}
\hline $\begin{array}{lllll}\text { Baseline demographics and disease } \\
\text { characteristics }\end{array}$ & \multicolumn{2}{l}{ IXE treatment arm } & & \multicolumn{2}{l}{ ADA treatment arm } \\
\cline { 2 - 3 } & $\begin{array}{l}\text { No MTX } \\
(\boldsymbol{N}=\mathbf{1 1 6})\end{array}$ & $\begin{array}{l}\text { MTX } \\
(\boldsymbol{N}=\mathbf{1 6 7})\end{array}$ & & $\begin{array}{l}\text { No MTX } \\
(\boldsymbol{N}=\mathbf{1 1 4})\end{array}$ & $\begin{array}{l}\text { MTX } \\
(\boldsymbol{N}=\mathbf{1 6 9})\end{array}$ \\
\hline BSA $\geq 10 \%, n(\%)$ & $47 / 116(40.5)$ & $66 / 167(39.5)$ & & $43 / 114(37.7)$ & $61 / 169(36.1)$ \\
Percentage BSA & $14.1(17.0)$ & $15.2(19.4)$ & & $12.7(14.4)$ & $13.0(16.4)$ \\
PASI total score & $8.3(8.3)$ & $7.6(9.1)$ & & $8.4(7.4)$ & $7.2(7.2)$ \\
DLQI total score & $11.6(8.1)$ & $8.5(6.9)$ & & $10.8(7.9)$ & $9.2(7.4)$ \\
NAPSI fingernails $>0, n(\%)$ & $83 / 116(71.6)$ & $108 / 167$ & & $76 / 114(66.7)$ & $101 / 168$ \\
& & $(64.7)$ & & $(60.1)$ \\
NAPSI fingernails & $\mathrm{d}$ & $22.1(20.9)$ & $17.8(16.2)$ & $20.1(18.3)$ & $18.4(14.6)$ \\
\hline
\end{tabular}

Unless indicated otherwise, data are presented as mean with the standard deviation (SD) in parentheses

$A D A$ Adalimumab, BSA body surface area, DLQI Dermatology Life Quality Index, HAQ-DI Health Assessment Questionnaire-Disability Index, IXE ixekizumab, $L D I-B$ Leeds Dactylitis Index-Basic, LEI Leeds Enthesitis Index, MTX methotrexate, $n$ number of patients in a group, $N$ number of patients, NAPSI Nail Psoriasis Area and Severity Index, PASI Psoriasis Area and Severity Index, $P s A$ psoriatic arthritis, SPARCC Spondyloarthritis Research Consortium of Canada, VAS visual analog scale

${ }^{a}$ Assessed in patients with SPARCC Enthesitis Index $>0$ at baseline

${ }^{b}$ Assessed in patients with LEI $>0$ at baseline

c Assessed in patients with LDI-B $>0$ at baseline

${ }^{d}$ Assessed in patients with NAPSI $>0$ at baseline

IXE (27.6\%) than with ADA (15.8\%). Among patients taking MTX, there were no significant differences in VLDA between IXE (20.4\%) and ADA (21.3\%) at week 52. Among patients not using MTX, significantly $(p=0.017)$ more patients achieved low disease activity or remission according to DAPSA $(\leq 14)$ at week 52 in the IXE (62.9\%) versus the ADA (46.5\%) group (ESM Table 1), whereas when used with MTX, there were no statistical differences between IXE and ADA in DAPSA remission $(\leq 4)$ (Table 2; Fig. 2c, d). There were no statistically significant differences in DAPSA $\leq 4$ (Table 2; Fig. 2c, d) and DAPSA $\leq 14$ responses at 52 weeks between treatment arms when used with MTX (ESM Table 1).

\section{Other Outcomes}

Resolution of enthesitis (SPARCC enthesitis $=0$ ) was similar with IXE and ADA regardless of whether they were used with or without MTX (Table 2). There were no statistically significant differences between IXE and ADA (with MTX/ without MTX) in SPARCC enthesitis Index $=0$ from week 8 through week 52 (Fig. 3a, b).

\section{Nail Psoriasis Outcome}

At week 52, IXE response on NAPSI fingernails $=0$ was similar regardless of whether it was used with MTX (66.7\%) or without MTX (68.7\%), while ADA response was numerically higher when used with MTX (64.4\%) than without MTX (51.3\%) (Table 2). For patients not taking MTX, significantly $(p=0.035)$ more patients achieved NAPSI $=0$ response with IXE $(68.7 \%)$ than with $\mathrm{ADA}(51.3 \%)$ at week 52 (Table 2).

\section{QoL Outcome and Physical Function}

Responses for QoL and function outcomes were lower with IXE when it was used with MTX than when used without MTX, while ADA responses were numerically higher when used with MTX than when used without MTX (Table 2). At week 52, for patients not taking MTX, HAQ-DI $\leq 0.5$ response was significantly 
Table 2 Efficacy and health outcomes at week 52

\begin{tabular}{|c|c|c|c|c|}
\hline \multirow[t]{2}{*}{ Efficacy and health outcomes ${ }^{a}$} & \multicolumn{2}{|l|}{ IXE treatment arm } & \multicolumn{2}{|l|}{ ADA treatment arm } \\
\hline & No MTX $(N=116)$ & $\operatorname{MTX}(N=167)$ & No MTX $(N=114)$ & $\operatorname{MTX}(N=169)$ \\
\hline \multicolumn{5}{|l|}{ Major endpoint } \\
\hline ACR50 + PASI 100 & $46 / 116(39.7)^{\dagger}$ & $65 / 167(38.9)$ & $23 / 114(20.2)$ & $51 / 169(30.2)$ \\
\hline ACR50 & $61 / 116(52.6)$ & $80 / 167(47.9)$ & $46 / 114(40.4)$ & $95 / 169(56.2)$ \\
\hline PASI100 & $76 / 116(65.5)^{+}$ & $106 / 167(63.5)^{*}$ & $42 / 114(36.8)$ & $75 / 169(44.4)$ \\
\hline \multicolumn{5}{|c|}{ PsA endpoints identified for treat-to-target } \\
\hline MDA & $56 / 116(48.3)^{*}$ & $78 / 167(46.7)$ & $37 / 114(32.5)$ & $79 / 169(46.7)$ \\
\hline $\mathrm{VLDA}^{\mathrm{b}}$ & $32 / 116(27.6)^{*}$ & $34 / 167(20.4)$ & $18 / 114(15.8)$ & $36 / 169(21.3)$ \\
\hline DAPSA remission $(\leq 4)$ & $41 / 116(35.3)$ & $44 / 167(26.3)$ & $27 / 114(23.7)$ & $53 / 169(31.4)$ \\
\hline \multicolumn{5}{|l|}{ Musculoskeletal endpoints } \\
\hline ACR20 & $83 / 116(71.6)$ & $114 / 167(68.3)$ & $68 / 114(59.6)$ & $127 / 169(75.1)$ \\
\hline ACR70 & $47 / 116(40.5)^{*}$ & $53 / 167(31.7)$ & $31 / 114(27.2)$ & $66 / 169(39.1)$ \\
\hline SPARCC Enthesitis Index $=0^{\mathrm{b}}$ & $41 / 78(52.6)$ & $66 / 111(59.5)$ & $34 / 76(44.7)$ & $49 / 95(51.6)$ \\
\hline \multicolumn{5}{|l|}{ Nail psoriasis endpoint } \\
\hline NAPSI fingernails $=0^{\mathrm{c}}$ & $57 / 83(68.7)^{*}$ & $72 / 108(66.7)$ & $39 / 76(51.3)$ & $65 / 101(64.4)$ \\
\hline \multicolumn{5}{|l|}{ Quality of life and function endpoint } \\
\hline HAQ-DI $\leq 0.5$ & $63 / 116(54.3)^{*}$ & $77 / 167(46.1)$ & $45 / 114(39.5)$ & $84 / 169(49.7)$ \\
\hline
\end{tabular}

Values are presented as $n / N$ with the percentage given in parentheses

ACR20/50/70 20, 50, 70\% improvement, respectively, in the American College of Rheumatology scale score, DAPSA Disease Activity in Psoriatic Arthritis, $M D A$ minimal disease activity, $n$ number of patients in a group, $N$ number of patients, PASI100 100\% improvement (= disease clearance) in the PASI score, $V L D A$ very low disease activity

${ }^{*},{ }^{\dagger},{ }^{\dagger}$ Significant difference IXE vs. ADA within the No MTX or MTX subgroups at: ${ }^{*} p<0.05,{ }^{\dagger} p \leq 0.01,{ }^{\dagger} p \leq 0.001$

${ }^{a}$ Post-hoc analyses of efficacy outcomes were performed in the intent-to-treat population. Missing data were imputed using non-responder imputation (NRI).

b Assessed for patients with SPARCC Enthesitis Index score $>0$ at baseline

c Assessed for patients with NAPSI fingernails score $>0$ at baseline

higher with IXE than with ADA $(p=0.026)$; statistically significant response was observed only at this timepoint.

\section{Safety}

\section{General Safety}

The overall safety of IXE and ADA was consistent with the known safety profile of both drugs (Table 3). The safety profile was consistent between subgroups who did or did not receive MTX for both IXE and ADA. Severe TEAEs, SAEs, and discontinuations due to AEs were numerically lower in the IXE treatment group than in the ADA treatment group regardless of whether patients received MTX or not. No deaths occurred during the study.

\section{Adverse Events of Special Interest}

Treatment-emergent adverse events of malignancies, MACE, IBD, injection site-reactions, depression, and neutropenia were generally similar in frequency with or without MTX for both treatment arms (IXE or ADA) (Table 3). 


\section{Without concomitant MTX}

a

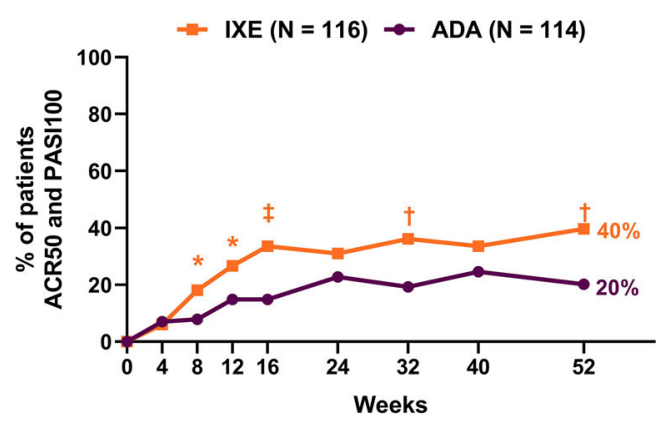

C

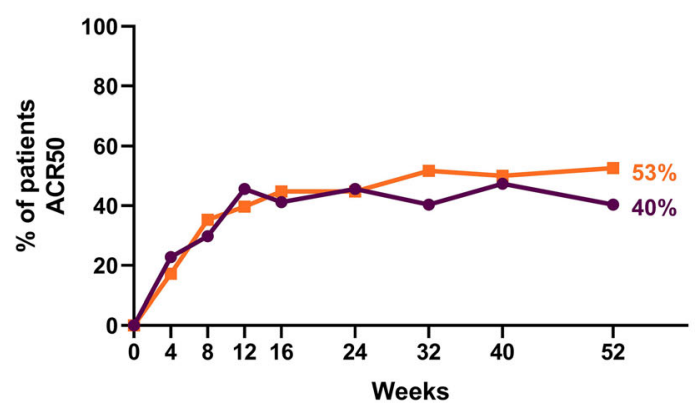

Fig. 1 Clinical response rates for major outcomes through week 52 in the intent-to-treat population (nonresponder imputation). a, b Percentage of patients achieving the combined ACR50 and PASI100 endpoints. c, d Percentage of patients achieving ACR50. ${ }^{*}, \dagger$ Significant difference IXE vs. $\mathrm{ADA}$ at: ${ }^{*} p<0.05$, and ${ }^{\dagger} p \leq 0.01$; ${ }^{*} p \leq 0.001$,

Numerically more abnormal liver function test values and cytopenia were reported with MTX than without MTX for both the IXE and ADA treatment arms. Numerically more infectious events were reported without MTX than with MTX.

\section{DISCUSSION}

The results from the SPIRIT-H2H study at 52 weeks suggested that the efficacy of IXE is independent of MTX in terms of achieving optimal outcomes in patients with PsA. In contrast, ADA efficacy was found to be numerically better with concomitant MTX use than without MTX use. A similar pattern of responses

\section{With Concomitant MTX}
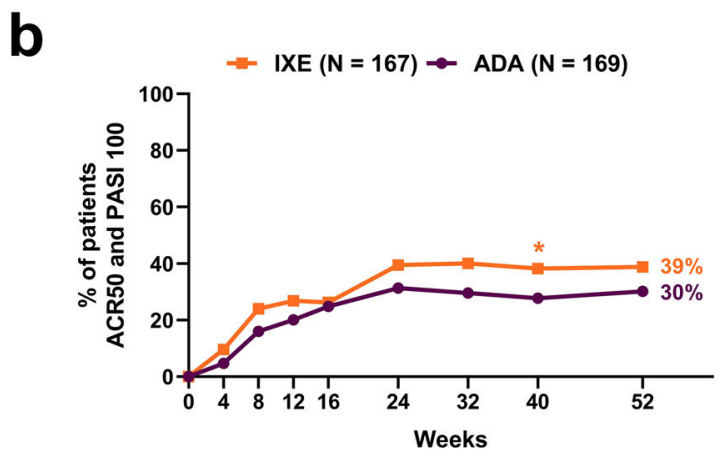

d

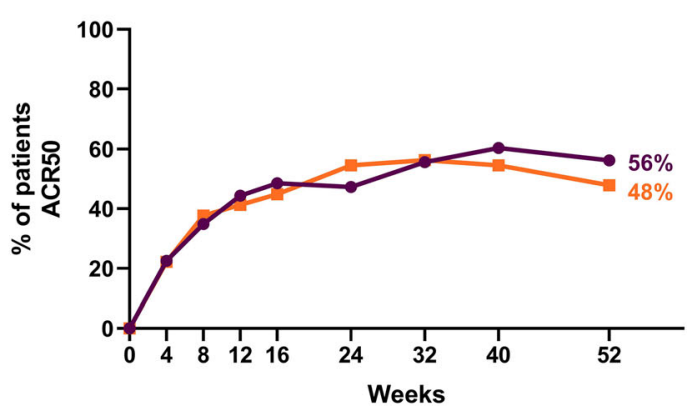

respectively. ACR50 50\% improvement in the American College of Rheumatology scale score, PASI100 100\% improvement (= disease clearance) in the Psoriasis Area and Severity Index score, $A D A$ adalimumab, IXE ixekizumab, $M T X$ methotrexate

was observed with IXE and ADA when used with all csDMARDs (MTX, leflunomide, sulfasalazine, or cyclosporine) [23]. These results are relevant in clinical practice for informing treatment decisions for patients with PsA for whom MTX may be not recommended (those who have inadequate response or intolerance to MTX) as ADA seems to be less effective than IXE for the treatment of clinical manifestations of PsA in terms of the outcomes in patients who are not taking concomitant MTX. Although EULAR acknowledges there is no clear evidence that MTX in combination with bDMARD is more efficacious than bDMARD monotherapy, the organization recommends using concomitant MTX with bDMARD in patients already on MTX without signs of intolerance [5]. The 


\section{Without concomitant MTX}

a

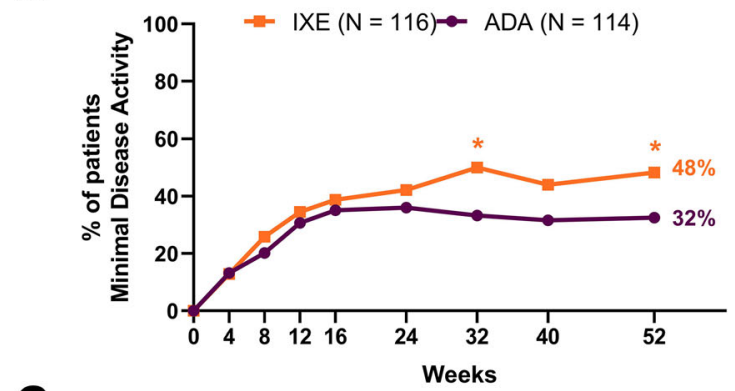

C

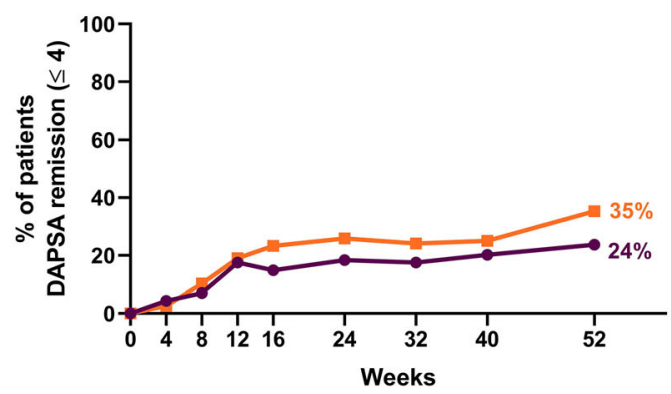

Fig. 2 Clinical response rates for psoriatic arthritis endpoints identified for treat-to-target through week 52 in the intent-to-treat population (nonresponder imputation). a, $\mathbf{b}$ Percentage of patients achieving minimal disease

findings from this analysis reported here can inform treatment decisions when considering concomitant MTX use with IXE or with ADA at initiation of treatment or for long-term maintenance.

The ADEPT study of ADA in patients with PsA found that ACR20, ACR50, and ACR70 responses were similar regardless of whether ADA was used with or without MTX [24, 25]. Our results showed ADA efficacy on treat-totarget endpoints (MDA and VLDA, as well as DAPSA low disease activity/remission), skin (PASI75/90/100), and QoL endpoints (DLQI $[0,1]$ ) was higher when used with MTX (Table 2; ESM Table 1). It has been shown that MTX in combination with ADA demonstrated better results on the PASI than ADA monotherapy in patients with moderate-to-severe PsO [26]. Since

\section{With Concomitant MTX}

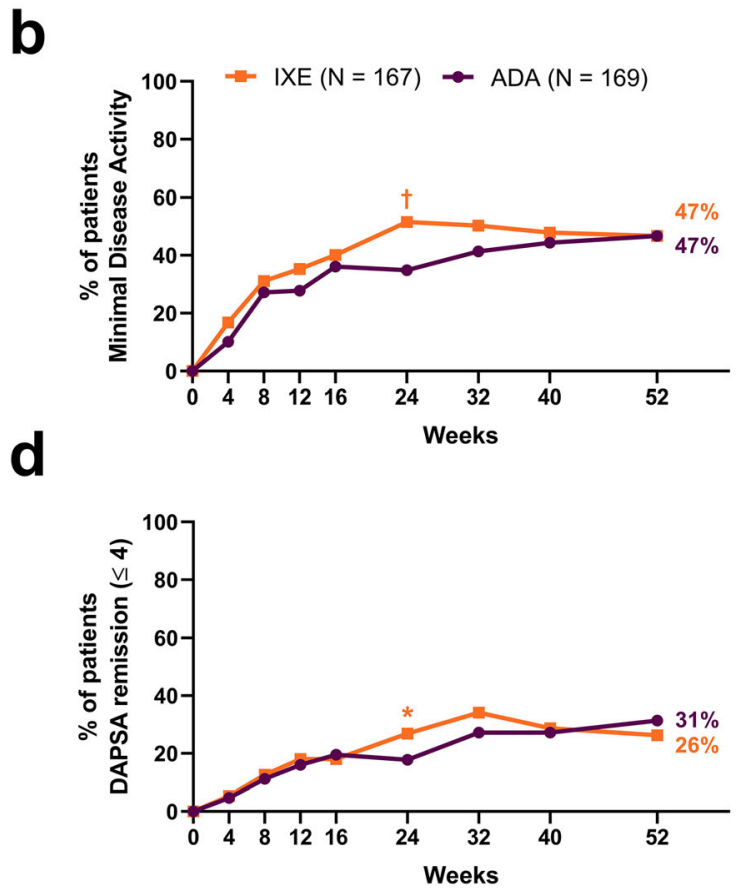

activity. c, d Percentage of patients achieving a DAPSA score $\leq 4$ (remission). ${ }^{*}{ }^{\dagger}$ 'Significant difference IXE vs. ADA at: ${ }^{*} p<0.05$ and ${ }^{\dagger} p \leq 0.01$. DAPSA Disease Activity in Psoriatic Arthritis

skin outcomes are a component of the MDA score and DLQI $(0,1)$, improvements in skin outcomes in the SPIRIT-H2H study with ADA in combination with MTX likely contributed to greater MDA and DLQI $(0,1)$ results. However, neither the ADEPT nor the SPIRIT-H2H study were designed to compare ADA as monotherapy with ADA plus concomitant MTX use as a primary endpoint. Both IXE and ADA are approved for the treatment of PsA with or without MTX $[21,27]$.

In this subanalysis of the SPIRIT-H2H trial comparing the effects of IXE to ADA in patients with PsA who were bDMARD-naïve, we demonstrated that response to IXE without MTX was higher than the response to ADA without MTX for multiple joint and skin endpoints. The EXCEED study comparing secuk- 


\section{Without concomitant MTX}

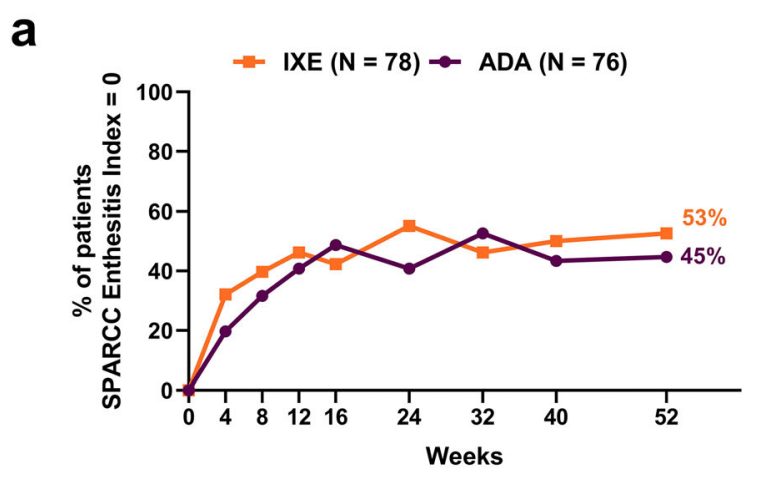

Fig. 3 Clinical response rates for a musculoskeletal outcome through week 52 in the intent-to-treat population (nonresponder imputation). a, b Percentage of patients achieving complete resolution in enthesitis according to the SPARCC scoring system (SPARCC =

inumab safety and efficacy versus ADA without concomitant csDMARD use in patients with active PsA who were naïve to biologic therapy and intolerant or with inadequate response to csDMARD [28] failed to show the superiority of secukinumab versus ADA on ACR20 (primary endpoint). However, EXCEED reported a significantly higher response with secukinumab versus ADA in some joint and skin endpoints. The SPIRIT-H2H and the EXCEED studies support the role of IL-17A inhibitors for the treatment of patients with PsA.

The main strength of this analysis is the study design with a 52-week duration, which allows the evaluation of efficacy of IXE and ADA with concomitant use of MTX over a long treatment duration. Since randomization was stratified by csDMARD use, the subgroups were well balanced, enabling proper comparisons. This study design permits the use of concomitant MTX therapy and better reflects clinical practice for patients with PsA. In an era of tailored medicine, this information adds to the importance of providing flexible treatment strategies depending on the patient's preference and tolerance to MTX. A limitation of this post-

\section{With Concomitant MTX}

b

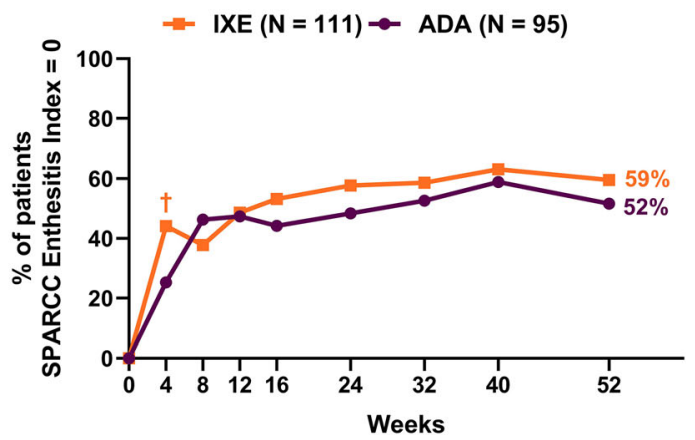

$0)$ in patients with SPARCC enthesitis index score $>0$ at baseline. ${ }^{\dagger}$ Significant difference IXE vs. ADA at $p \leq 0.01$. $S P A R C C$ Spondyloarthritis Research Consortium of Canada index

hoc analysis is the fact the SPIRIT-H2H study was not powered to evaluate the benefit of MTX in combination with a bDMARD, resulting in small sample sizes in each subgroup. The openlabel study design increases the real-world clinical setting compared to double-blind trials. The double-blind study design would have increased the burden due to the number of injections per week and make the study not acceptable for patients. While the open-label design could have influenced patients' assessment outcomes, such as components of ACR measure, in the SPIRIT-H2H study, patients were treated equally regardless of which treatment they received, thereby minimizing the bias of patients' assessment outcomes.

Although the majority of patients enrolled in this study had polyarthritis, a similar finding was observed in other clinical trials in patients with PsA. Enrolled patients may not fully represent all PsA patients in daily real-world clinical practice (e.g., patients in this study predominantly had polyarthritis while in clinical practice patients mostly have oligoarticular involvement). 
Table 3 Safety outcomes at week 52

\begin{tabular}{|c|c|c|c|c|}
\hline \multirow[t]{2}{*}{ Safety outcomes } & \multicolumn{2}{|c|}{ IXE treatment arm } & \multicolumn{2}{|c|}{ ADA treatment arm } \\
\hline & $\begin{array}{l}\text { No MTX } \\
(N=116)\end{array}$ & $\begin{array}{l}\text { MTX } \\
(N=167)\end{array}$ & $\begin{array}{l}\text { No MTX } \\
(N=114)\end{array}$ & $\begin{array}{l}\text { MTX } \\
(N=169)\end{array}$ \\
\hline $\begin{array}{l}\text { Treatment-emergent adverse } \\
\quad \text { events } \geq 1\end{array}$ & $92(79.3)$ & $117(70.1)$ & $80(70.2)$ & $114(67.5)$ \\
\hline Mild & $43(37.1)$ & $52(31.1)$ & $31(27.2)$ & $54(32.0)$ \\
\hline Moderate & $44(37.9)$ & $61(36.5)$ & $39(34.2)$ & $50(29.6)$ \\
\hline Severe $^{\mathrm{a}}$ & $5(4.3)$ & $4(2.4)$ & $10(8.8)$ & $10(5.9)$ \\
\hline Serious adverse events ${ }^{\mathrm{b}}$ & $8(6.9)$ & $4(2.4)$ & $17(14.9)$ & $18(10.7)$ \\
\hline Deaths & 0 & 0 & 0 & 0 \\
\hline $\begin{array}{l}\text { Discontinuations due to adverse } \\
\text { events }\end{array}$ & $8(6.9)$ & $4(2.4)$ & $9(7.9)$ & $12(7.1)$ \\
\hline \multicolumn{5}{|l|}{ Adverse events of special interest } \\
\hline Infections & $54(46.6)$ & $65(38.9)$ & $49(43.0)$ & $62(36.7)$ \\
\hline Nasopharyngitis & $21(18.1)$ & $17(10.2)$ & $12(10.5)$ & $11(6.5)$ \\
\hline Upper respiratory tract infection & $5(4.3)$ & $13(7.8)$ & $7(6.1)$ & $11(6.5)$ \\
\hline Serious infections & $4(3.4)$ & $1(0.6)$ & $5(4.4)$ & $3(1.8)$ \\
\hline Malignancies & 0 & 0 & $1(0.9)$ & $3(1.8)$ \\
\hline MACE & 0 & 0 & $1(0.9)$ & $1(0.6)$ \\
\hline $\mathrm{IBD}^{\mathrm{c}}$ & $1(0.9)$ & $1(0.6)$ & 0 & 0 \\
\hline Injection site reactions & $13(11.2)$ & $17(10.2)$ & $3(2.6)$ & $7(4.1)$ \\
\hline Depression & $4(3.4)$ & $1(0.6)$ & $5(4.4)$ & $4(2.4)$ \\
\hline \multicolumn{5}{|l|}{ Liver function test values ${ }^{\mathrm{d}}$} \\
\hline ALT elevation $^{\mathrm{e}}$ & $1(0.4)$ & $2(0.7)$ & $2(0.7)$ & $9(3.2)$ \\
\hline AST elevation ${ }^{e}$ & $1(0.4)$ & $1(0.4)$ & $1(0.4)$ & $6(2.1)$ \\
\hline Total bilirubin ${ }^{f}$ & 0 & $3(1.1)$ & 0 & $2(0.7)$ \\
\hline $\operatorname{ALP}^{f}$ & $1(0.4)$ & $4(1.4)$ & $1(0.4)$ & $4(1.4)$ \\
\hline Cytopenias & $3(2.6)$ & $6(3.6)$ & $3(2.6)$ & $9(5.3)$ \\
\hline Neutropenia & $2(1.7)$ & $2(1.2)$ & $1(0.9)$ & $4(2.4)$ \\
\hline
\end{tabular}

Unless otherwise indicated, values are presented as a number with the percentage $(n / N)$ given in parentheses

$A L P$ Alkaline phosphatase, $A L T$ alanine aminotransferase, $A S T$ aspartate aminotransferase, IBD inflammatory bowel disease, $M A C E$ major adverse cardiovascular events, $n$ number of patients in a group, $N$ number of patients, $U L N$ upper limit normal from performed laboratoy reference ranges

a Severity of an adverse event is judged by patient or investigator

b Serious adverse event met the serious criteria of the International Council for Harmonization

c Two IBD events were reported in the IXE treatment group during the 0 - to 24 -week period but only 1 case was adjudicated as confirmed Crohn's disease. One event was reported as colitis ulcerative but was not adjudicated as confirmed ulcerative colitis [21]

${ }^{d}$ Liver function test values were calculated as percentages using the denominator total of 283 for both the IXE and ADA groups

Post-baseline $\geq 3$ ULN

f Post-baseline bilirubin $\geq 1.5$ ULN; post-baseline ALP $>1.5$ ULN 


\section{CONCLUSION}

Ixekizumab demonstrated similar efficacy across different domains of PsA regardless of whether it was used with or without MTX. As a clinical issue for rheumatologists, this raises the possibility that it may not be necessary to treat with patients currently taking or about to start IXE with concurrent MTX. In patients who did not receive MTX, IXE long-term efficacy was greater than that of ADA across multiple skin and musculoskeletal disease-specific outcomes. The safety of both agents was consistent with their known safety profile regardless of concomitant MTX use. The findings of this study increase awareness of current treatment options and inform evidence-based treatment decisions when considering concomitant MTX use when prescribing IXE or ADA for patients with PsA and active psoriatic skin disease.

\section{ACKNOWLEDGEMENTS}

The authors thank the study participants, caregivers, and investigators.

Funding. This study and the journal's Rapid Service Fee were funded by Eli Lilly and Company, which contributed to study design, data collection, data analysis, data interpretation, manuscript preparation, and publication decisions.

Medical Writing and Editorial Assistance. Elsa Mevel, PhD and Cynthia R. Abbott who are employees of Eli Lilly provided writing and editorial assistance. Funding for this assistance was provided by Eli Lilly and Company.

Authorship. All named authors meet the International Committee of Medical Journal Editors (ICMJE) criteria for authorship for this article, had full access to all the data in this study, take responsibility for the integrity of data and accuracy of the data analysis, and have given their approval for this version to be published.
Disclosures. Josef S Smolen reports research grants from AbbVie, Astra-Zeneca, Janssen, Lilly, Merck Sharp \& Dohme, Novartis, Pfizer, and Roche; and personal fees from AbbVie, Amgen, Astra-Zeneca, Astro, Bristol-Myers Squibb, Celgene, Celltrion, Chugai, Gilead, ILTOO, Janssen, Lilly, MSD, Novartis-Sandoz, Pfizer, Roche, Samsung, Sanofi, and UCB. Anthony Sebba is a consultant for Eli Lilly and Company, Genentech, Gilead, Regeneron Pharmaceuticals, Sanofi, and is on the speaker bureau of Eli Lilly and Company, Roche, and Sanofi. Eric M Ruderman is a consultant for AbbVie, Amgen, Bristol-Meyers Squibb, Eli Lilly and Company, Gilead, Janssen, Novartis, and Pfizer. Hendrik Schulze-Koops has research grants from AbbVie, Novartis, and has received honoraria for consultancies and/or speaking engagements from AbbVie, Amgen, Bristol-Myers Squibb, Celgene, Celltrion, Chugai, Gilead, Janssen, Eli Lilly and Company, Merck Sharp \& Dohme, Novartis-Sandoz, Pfizer, Roche, Sanofi. Christophe Sapin, Amanda M Gellett, Aubrey Trevelin Sprabery, Soyi Liu-Leage, Sreekumar Pillai, Lingnan Li, Inmaculada de la Torre, Gaia Gallo and Paulo Reis are employees and shareholders at Eli Lilly and Company. Peter Nash has received grant/research support from AbbVie, Bristol-Myers Squibb, Celgene, Eli Lilly and Company, Gilead, Janssen, MSD, Novartis, Pfizer, Roche, Sanofi and UCB, is a consultant for AbbVie, Bristol-Myers Squibb, Celgene, Eli Lilly and Company, Gilead, Janssen, Merck Sharp \& Dohme, Novartis, Pfizer, Roche, Sanofi, UCB, and is on the speakers bureau of AbbVie, BristolMyers Squibb, Celgene, Eli Lilly and Company, Gilead, Janssen, Merck Sharp \& Dohme, Novartis, Pfizer Inc, Roche, Sanofi, and UC.

Compliance with Ethics Guidelines. All procedures performed in studies involving human participants were in accordance with the ethical standards of the institutional and/or national research committees at all sites where these studies were conducted and with the 1964 Helsinki declaration and its later amendments or comparable ethical standards. Informed consent was obtained from all individual participants included in the studies. The study protocol was approved by the ethical review 
board of all participating centers prior to the start of study-related procedures, see Supplementary Material for the full list of ethics committees. The main ethics committee was Whipps Cross University Hospital, London, United Kingdom (registration \# 17/LO/0794).

Data Availability. The datasets generated and/or analyzed during the current study are available from the corresponding author on reasonable request.

Open Access. This article is licensed under a Creative Commons Attribution-NonCommercial 4.0 International License, which permits any non-commercial use, sharing, adaptation, distribution and reproduction in any medium or format, as long as you give appropriate credit to the original author(s) and the source, provide a link to the Creative Commons licence, and indicate if changes were made. The images or other third party material in this article are included in the article's Creative Commons licence, unless indicated otherwise in a credit line to the material. If material is not included in the article's Creative Commons licence and your intended use is not permitted by statutory regulation or exceeds the permitted use, you will need to obtain permission directly from the copyright holder. To view a copy of this licence, visit http://creativecommons.org/licenses/by$\mathrm{nc} / 4.0 /$.

\section{REFERENCES}

1. Coates LC, Helliwell PS. Psoriatic arthritis: state of the art review. Clin Med. 2017;17(1):65-70.

2. Ritchlin CT, Colbert RA, Gladman DD. Psoriatic arthritis. N Engl J Med. 2017;376(10):957-70.

3. Gladman DD, Antoni C, Mease P, et al. Psoriatic arthritis: epidemiology, clinical features, course, and outcome. Ann Rheum Dis. 2005;64(Suppl 2): ii14-7.

4. Wilsdon TD, Whittle SL, Thynne TR, et al. Methotrexate for psoriatic arthritis. Cochrane Database Syst Rev. 2019;1:CD012722.
5. Gossec L, Baraliakos X, Kerschbaumer A, et al. EULAR recommendations for the management of psoriatic arthritis with pharmacological therapies: 2019 update. Ann Rheum Dis. 2020;79(6):700-12.

6. Gossec L, Smolen JS, Ramiro S, et al. European League Against Rheumatism (EULAR) recommendations for the management of psoriatic arthritis with pharmacological therapies: 2015 update. Ann Rheum Dis. 2016;75(3):499-510.

7. Ye W, Coates LC. Should methotrexate have any place in the treatment of psoriatic arthritis? Rheum Dis Clin North Am. 2019;45(3):325-39.

8. Coates LC, Moverley AR, McParland L, et al. Effect of tight control of inflammation in early psoriatic arthritis (TICOPA): a UK multicentre, open-label, randomised controlled trial. Lancet. 2015;386(10012):2489-98.

9. Mease PJ, Gladman DD, Collier DH, et al. Etanercept and methotrexate as monotherapy or in combination for psoriatic arthritis: primary results from a randomized, controlled phase III trial. Arthritis Rheumatol. 2019;71(7):1112-24.

10. Kerschbaumer A, Smolen JS, Aletaha D, et al. Is methotrexate as efficacious as etanercept in psoriatic arthritis patients? Comment on the article by Mease et al. Arthritis Rheumatol. 2020;72(7): 1227-9.

11. Behrens F, Canete JD, Olivieri I, et al. Tumour necrosis factor inhibitor monotherapy vs combination with MTX in the treatment of PsA: a systematic review of the literature. Rheumatology. 2015;54(5):915-26.

12. Behrens F, Koehm M, Arndt U, et al. Does concomitant methotrexate with adalimumab influence treatment outcomes in patients with psoriatic arthritis? Data from a large observational study. J Rheumatol. 2016;43(3):632-9.

13. van der Heijde D, Klareskog L, Rodriguez-Valverde $\mathrm{V}$, et al. Comparison of etanercept and methotrexate, alone and combined, in the treatment of rheumatoid arthritis: two-year clinical and radiographic results from the TEMPO study, a doubleblind, randomized trial. Arthritis Rheum. 2006;54(4):1063-74.

14. Breedveld FC, Weisman MH, Kavanaugh AF, et al. The PREMIER study: a multicenter, randomized, double-blind clinical trial of combination therapy with adalimumab plus methotrexate versus methotrexate alone or adalimumab alone in patients with early, aggressive rheumatoid arthritis who had not had previous methotrexate treatment. Arthritis Rheum. 2006;54(1):26-37. 
15. Smolen JS, Kay J, Doyle MK, et al. Golimumab in patients with active rheumatoid arthritis after treatment with tumour necrosis factor alpha inhibitors (GO-AFTER study): a multicentre, randomised, double-blind, placebo-controlled, phase III trial. Lancet. 2009;374(9685):210-21.

16. Kaneko Y, Kato M, Tanaka Y, et al. Tocilizumab discontinuation after attaining remission in patients with rheumatoid arthritis who were treated with tocilizumab alone or in combination with methotrexate: results from a prospective randomised controlled study (the second year of the SURPRISE study). Ann Rheum Dis. 2018;77(9): 1268-75.

17. Dougados M, Kissel K, Conaghan PG, et al. Clinical, radiographic and immunogenic effects after 1 year of tocilizumab-based treatment strategies in rheumatoid arthritis: the ACT-RAY study. Ann Rheum Dis. 2014;73(5):803-9.

18. Liu L, Lu J, Allan BW, et al. Generation and characterization of ixekizumab, a humanized monoclonal antibody that neutralizes interleukin-17A. J Inflamm Res. 2016;9:39-50.

19. Toussirot E. Ixekizumab: an anti- IL-17A monoclonal antibody for the treatment of psoriatic arthritis. Expert Opin Biol Ther. 2018;18(1):101-7.

20. Paller AS, Seyger MM, Magariños G, et al. Efficacy and safety of ixekizumab in a phase 3 , randomized, double-blind, placebo-controlled study in paediatric patients with moderate-to-severe plaque psoriasis (IXORA-PEDS) Br J Dermatol. 2020;183(2): 231-41.

21. Eli Lilly and Company. Highlights of prescribing information-Taltz. 2020. https://uspl.lilly.com/ taltz/taltz.html\#pi.

22. Mease PJ, Smolen JS, Behrens F, et al. A head-tohead comparison of the efficacy and safety of ixekizumab and adalimumab in biological-naive patients with active psoriatic arthritis: 24-week results of a randomised, open-label, blinded-assessor trial. Ann Rheum Dis. 2020;79(1):123-31.

23. Smolen JS, Mease $\mathrm{P}$, Tahir $\mathrm{H}$, et al. Multicentre, randomised, open-label, parallel-group study evaluating the efficacy and safety of ixekizumab versus adalimumab in patients with psoriatic arthritis naive to biological disease-modifying antirheumatic drug: final results by week 52 . Ann Rheum Dis. 2020;79:1310-9.

24. Mease PJ, Gladman DD, Ritchlin CT, et al. Adalimumab for the treatment of patients with moderately to severely active psoriatic arthritis: results of a double-blind, randomized, placebo-controlled trial. Arthritis Rheum. 2005;52(10):3279-89.

25. Gladman DD, Mease PJ, Ritchlin CT, et al. Adalimumab for long-term treatment of psoriatic arthritis: forty-eight week data from the adalimumab effectiveness in psoriatic arthritis trial. Arthritis Rheum. 2007;56(2):476-88.

26. van den Reek JM, van Lumig PP, Kievit W, et al. Effectiveness of adalimumab dose escalation, combination therapy of adalimumab with methotrexate, or both in patients with psoriasis in daily practice. J Dermatolog Treat. 2013;24(5):361-8.

27. AbbVie Inc. Highlights of prescribing information-Humira. 2020. https://www.rxabbvie.com/ pdf/humira.pdf.

28. McInnes IB, Behrens F, Mease PJ, et al. Secukinumab versus adalimumab for treatment of active psoriatic arthritis (EXCEED): a double-blind, parallel-group, randomised, active-controlled, phase $3 \mathrm{~b}$ trial. Lancet. 2020;395(10235):1496-505. 